\title{
An Improved Protocol for Biginelli Reaction
}

\author{
Vivek Srivastava \\ Applied Science, NIIT University, Neemrana, India \\ Email: vivek.srivastava.chem@gmail.com
}

Received February 12, 2013; revised March 15, 2013; accepted March 28, 2013

Copyright (C) 2013 Vivek Srivastava. This is an open access article distributed under the Creative Commons Attribution License, which permits unrestricted use, distribution, and reproduction in any medium, provided the original work is properly cited.

\begin{abstract}
[Hmim][Tfa] was tested as catalyst for the Biginelli reaction multicomponent reaction under conventional and non conventional heat source i.e. Microwave heating. Low catalyst loading, reduced reaction time and operational simplicity are the main highlights of this proposed protocol. Using our proposed protocol, we tested 30 different biologically active compounds in good yield.
\end{abstract}

Keywords: [Hmim][Tfa]; Biginelli Reaction; Dihydropyrimidines

\section{Introduction}

Multicomponent reactions (MCRs) are highely important reaction for organic chemists, especially in medicinal chemistry feild. [1,2]. Biginelli reaction is one of the most studied reaction in the area of multicomponent reactions [2] and dihydropyrimidin-2(1H)-ones (DHPMs) by the condensation of aldehyde, $\beta$-keto ester and urea in the presence of acid [3]. These 3,4-Dihydropyrimidin$2(1 \mathrm{H})$-ones are useful in the synthesis of pharmacological activity compounds [4].

Various catalysts have been used to catalyze this reaction like protic acids [5], Lewis acids [6], heterogeneous acidic catalyst [7] and reagents like iodine [8], NBS [9] etc. But many of these protocols are still suffering from different drawbacks such as longer reaction time, high catalyst loading, expensive reagents, corrosive reagents, low yields of products, and large amounts of solid supports which would eventually result in the production of large amounts of toxic waste [10] by considering these facts, we focused our study to task specific ionic liquid and Microwave heating.

The use of ionic liquids (ILs) in chemical research has gained considerable interest from the last decades [11-15]. ILs can be easily modified due to their tunable physicochemical properties according to the needs of the reaction e.g. for specific reactions, extractions or separations, and are then referred to as "task specific ionic liquids” (TSILs) [16].

The use of microwave (MW) irradiation is the alternative heating technique in synthetic chemistry [17]. Various group reviewed ionic liquid/MW combination of dif- ferent types of organic transformations like cycloaddition reaction, Heck reaction, Aza-Michael addition, Epoxidation, Fisher esterification reaction, Halogenation reactions, Dehalogenation reactions etc $[18,19]$.

Our study is mainly focused on to develop a new and more environmentally benign protocol for Biginelli reaction as well as to synthesize the biologically active compounds using task specific ionic liquids under microwave irradiation [20,21].

\section{Experimental Procedure}

All the chemicals were purchased from SD fine chemicals, Acros. NMR spectra were recorded on standard Bruker 300WB spectrometer with an Avance console at 300 and $75 \mathrm{MHz}$ for ${ }^{1} \mathrm{H}$ and ${ }^{13} \mathrm{C}$ NMR respectively. All the ILs was prepared according to the procedure reported in literature [6,7,20-22]. The microwave reactions were carried out using BPL Sanyo (India), mono-made, multi power; power source: $230 \mathrm{~V}, 50 \mathrm{~Hz}$, microwave frequency: $2450 \mathrm{MHz}$ microwave oven.

General procedure for preparation of DHPMs 1-10

A mixture of aldehyde $(1 \mathrm{mmol}), \beta$-keto ester $(1 \mathrm{mmol})$ and ammonia source $(1.5 \mathrm{mmol})$ were mixed with [Hmim][Tfa] $(0.1 \mathrm{mmol})$ and placed in a $50 \mathrm{~mL}$ conical flask. The flask was exposed to MW heating for $2 \mathrm{~min}$ at $630 \mathrm{~W}$ or the whole reaction mixture was heated at $100^{\circ} \mathrm{C}$ in a round bottom flask. After the completion the reaction (monitored by TLC: eluent: n-hexane/ethyl acetate: $3 / 1$ ), cold water was added and the precipitated product was separated by simple filtration. If needed, the crude product was purified by column chromatography or re-crystallization method using ethyl acetate/n-hexane 
or ethanol as eluent.

\section{Results and Discussion}

We examined four different types of ionic liquid such as $[\mathrm{Hmim}]\left[\mathrm{HSO}_{4}\right],[\mathrm{Hmim}][\mathrm{Tfa}],[\mathrm{Bmim}]\left[\mathrm{BF}_{4}\right]$ and $[\mathrm{Bmim}]$ $\left[\mathrm{PF}_{6}\right]$ for the synthesis of DHPMs under microwave irradiation for our model Biginelli reaction with benzaldehyde, ethylacetoacetate and urea under MW irradiation as well as thermal heating. The results are summaries in

\section{Table 1.}

As per Table 1, [Hmim][Tfa] was found highly active in term of yield and reaction time under MW heating over conventional heating and the reaction product i.e 3,4-dihydropyrimidin-2 (1H)-ones 4 was obtained in good yield. After getting delightful results, a variety of aromatic aldehydes, 1,3-carbonyl compounds, urea or thiourea underwent three-component condensation smoothly (Scheme 1) were tested and the corresponding reaction products are summarised in Table 2.

Table 1. Optimization for the reaction condition of Biginelli reaction.

\begin{tabular}{|c|c|c|c|c|}
\hline S. No. & Catalysts (0.1 mmol) & Types of heating & Time (min.) & Yield (\%) \\
\hline 1 & {$[\mathrm{Hmim}]\left[\mathrm{HSO}_{4}\right]$} & $100^{\circ} \mathrm{C}$ & 120 & 85 \\
\hline 2 & [Hmim] $[\mathrm{Tfa}]$ & $100^{\circ} \mathrm{C}$ & 120 & 95 \\
\hline 3 & {$[\mathrm{Bmim}]\left[\mathrm{BF}_{4}\right]$} & $100^{\circ} \mathrm{C}$ & 120 & 36 \\
\hline 4 & {$[\mathrm{Bmim}]\left[\mathrm{PF}_{6}\right]$} & $100^{\circ} \mathrm{C}$ & 120 & 30 \\
\hline 5 & $\mathrm{H}_{2} \mathrm{SO}_{4}$ & $100^{\circ} \mathrm{C}$ & 120 & 65 \\
\hline 6 & $\mathrm{HCl}$ & $100^{\circ} \mathrm{C}$ & 120 & 60 \\
\hline 7 & K-10 clay & $100^{\circ} \mathrm{C}$ & 120 & 48 \\
\hline 8 & Iodine & $100^{\circ} \mathrm{C}$ & 120 & 45 \\
\hline 9 & PS-PEG-SO ${ }_{3} \mathrm{H}$ & $100^{\circ} \mathrm{C}$ & 120 & 39 \\
\hline 10 & $\begin{array}{l}\text { trifluoromethane } \\
\text { sulfonic acid }\end{array}$ & $100^{\circ} \mathrm{C}$ & 120 & 50 \\
\hline 11 & $\begin{array}{c}\text { phosphinite ionic } \\
\text { liquid }\end{array}$ & $100^{\circ} \mathrm{C}$ & 120 & 62 \\
\hline 12 & L-Proline & $100^{\circ} \mathrm{C}$ & 120 & - \\
\hline 13 & L-Proline & r.t & 120 & 15 \\
\hline 14 & {$[\mathrm{Hmim}]\left[\mathrm{HSO}_{4}\right]$} & $630 \mathrm{~W}$ & 2 & 95 \\
\hline 15 & {$[\mathrm{Hmim}][\mathrm{Tfa}]$} & $630 \mathrm{~W}$ & 2 & 99 \\
\hline 16 & {$[\mathrm{Bmim}]\left[\mathrm{BF}_{4}\right]$} & $630 \mathrm{~W}$ & 2 & 58 \\
\hline 17 & {$[\mathrm{Bmim}]\left[\mathrm{PF}_{6}\right]$} & $630 \mathrm{~W}$ & 2 & 55 \\
\hline 18 & $\begin{array}{c}{[\mathrm{Hmim}][\mathrm{Tfa}]} \\
(0.5 \mathrm{mmol})\end{array}$ & $630 \mathrm{~W}$ & 2 & 99 \\
\hline 19 & $\begin{array}{l}{[\mathrm{Hmim}][\mathrm{Tfa}]} \\
(0.05 \mathrm{mmol})\end{array}$ & $630 \mathrm{~W}$ & 2 & 85 \\
\hline 20 & $\begin{array}{c}{[\text { Hmim] }[\mathrm{Tfa}]} \\
(0.05 \mathrm{mmol})\end{array}$ & $630 \mathrm{~W}$ & r.t & 85 \\
\hline
\end{tabular}

*isolated yield.

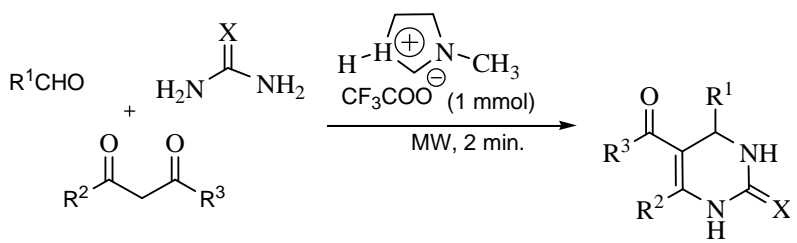

Scheme 1.

Table 2. Synthesis of DHPMs.

\begin{tabular}{|c|c|c|c|c|c|}
\hline Entry & $\mathrm{R}^{1}$ & $\mathrm{R}^{2}$ & $\mathrm{R}^{3}$ & $\mathrm{X}$ & Yield $^{\text {ab }}$ \\
\hline 1 & $\mathrm{C}_{6} \mathrm{H}_{5}$ & $\mathrm{Me}$ & OEt & $\mathrm{O}$ & 92 \\
\hline 2 & $4-\mathrm{MeOC}_{6} \mathrm{H}_{4}$ & $\mathrm{Me}$ & OEt & $\mathrm{O}$ & 94 \\
\hline 3 & $4-\mathrm{ClC}_{6} \mathrm{H}_{4}$ & $\mathrm{Me}$ & OEt & $\mathrm{O}$ & 90 \\
\hline 4 & $4-\mathrm{NO}_{2} \mathrm{C}_{6} \mathrm{H}_{4}$ & $\mathrm{Me}$ & OEt & $\mathrm{O}$ & 93 \\
\hline 5 & $3-\mathrm{NO}_{2} \mathrm{C}_{6} \mathrm{H}_{4}$ & $\mathrm{Me}$ & OEt & $\mathrm{O}$ & 90 \\
\hline 6 & $\mathrm{C}_{6} \mathrm{H}_{5}$ & $\mathrm{Me}$ & OMe & $\mathrm{O}$ & 95 \\
\hline 7 & $4-\mathrm{MeOC}_{6} \mathrm{H}_{4}$ & $\mathrm{Me}$ & OMe & $\mathrm{O}$ & 89 \\
\hline 8 & 4-OH-3-OMeC ${ }_{6} \mathrm{H}_{3}$ & $\mathrm{Me}$ & OMe & $\mathrm{O}$ & 95 \\
\hline 9 & $4-\mathrm{MeOC}_{6} \mathrm{H}_{4}$ & $\mathrm{Me}$ & OEt & $\mathrm{S}$ & 95 \\
\hline 10 & $4-\mathrm{ClC}_{6} \mathrm{H}_{4}$ & $\mathrm{Me}$ & OEt & $\mathrm{S}$ & 94 \\
\hline 11 & $2-\mathrm{ClC}_{6} \mathrm{H}_{4}$ & Et & OMe & $\mathrm{S}$ & 82 \\
\hline 12 & $3-\mathrm{ClC}_{6} \mathrm{H}_{4}$ & Et & $\mathrm{OMe}$ & $\mathrm{S}$ & 89 \\
\hline 13 & $4-\mathrm{ClC}_{6} \mathrm{H}_{4}$ & Et & OMe & $\mathrm{S}$ & 79 \\
\hline 14 & 3- $\mathrm{BrC}_{6} \mathrm{H}_{4}$ & Et & OMe & $\mathrm{S}$ & 90 \\
\hline 15 & $4-\mathrm{BrC}_{6} \mathrm{H}_{4}$ & Et & OMe & $\mathrm{S}$ & 91 \\
\hline 16 & $2-\mathrm{CH}_{3} \mathrm{C}_{6} \mathrm{H}_{4}$ & $\mathrm{Me}$ & OMe & $\mathrm{S}$ & 92 \\
\hline 17 & $3-\mathrm{CH}_{3} \mathrm{C}_{6} \mathrm{H}_{4}$ & $\mathrm{Me}$ & OMe & $\mathrm{S}$ & 90 \\
\hline 18 & 4- $\mathrm{CH}_{3} \mathrm{C}_{6} \mathrm{H}_{4}$ & $\mathrm{Me}$ & OMe & $\mathrm{S}$ & 93 \\
\hline 19 & $2-\mathrm{OCH}_{3} \mathrm{C}_{6} \mathrm{H}_{4}$ & $\mathrm{Me}$ & OMe & $\mathrm{S}$ & 91 \\
\hline 20 & $2-\mathrm{NO}_{2} \mathrm{C}_{6} \mathrm{H}_{4}$ & $\mathrm{Me}$ & OMe & $\mathrm{S}$ & 90 \\
\hline 21 & $3-\mathrm{NO}_{2} \mathrm{C}_{6} \mathrm{H}_{4}$ & $\mathrm{Me}$ & OMe & $\mathrm{S}$ & 85 \\
\hline 22 & $2-\mathrm{FC}_{6} \mathrm{H}_{4}$ & $\mathrm{Me}$ & OMe & $\mathrm{S}$ & 84 \\
\hline 23 & $3-\mathrm{FC}_{6} \mathrm{H}_{4}$ & Et & OMe & $\mathrm{S}$ & 89 \\
\hline 24 & $2-\mathrm{OC}_{2} \mathrm{H}_{5} \mathrm{C}_{6} \mathrm{H}_{4}$ & Et & OMe & $\mathrm{S}$ & 91 \\
\hline 25 & 2-OH-5- $\mathrm{BrC}_{6} \mathrm{H}_{3}$ & Et & OMe & $\mathrm{S}$ & 92 \\
\hline 26 & $3-\mathrm{NO}_{2} \mathrm{C}_{6} \mathrm{H}_{4}$ & $\mathrm{Me}$ & $\mathrm{CH}_{3} \mathrm{NH}$ & $\mathrm{O}$ & 93 \\
\hline 27 & $3-\mathrm{NO}_{2} \mathrm{C}_{6} \mathrm{H}_{4}$ & $\mathrm{Me}$ & $\mathrm{CH}_{3} \mathrm{NH}$ & $\mathrm{S}$ & 94 \\
\hline 28 & 4- $\mathrm{ClC}_{6} \mathrm{H}_{4}$ & $\mathrm{Me}$ & $\left(\mathrm{CH}_{3}\right)_{2} \mathrm{O}$ & $\mathrm{O}$ & 92 \\
\hline 29 & $4-\mathrm{NO}_{2} \mathrm{C}_{6} \mathrm{H}_{4}$ & $\mathrm{Me}$ & $\left(\mathrm{CH}_{3}\right)_{2} \mathrm{O}$ & $\mathrm{O}$ & 93 \\
\hline 30 & $4-\mathrm{FC}_{6} \mathrm{H}_{4}$ & $\mathrm{Me}$ & $\left(\mathrm{CH}_{3}\right)_{2} \mathrm{O}$ & $\mathrm{O}$ & 89 \\
\hline
\end{tabular}

${ }^{\mathrm{a}}$ Isolated yield; ${ }^{\mathrm{b}}$ The reaction products were characterized by ${ }^{1} \mathrm{HNMR}$ and found similar to reported data ${ }^{20-22}$.

The aromatic aldehydes carrying either electron dona- 
ting or electron-withdrawing substituents reacted well under the reaction conditions to give the corresponding DHPMs in high yield. Thiourea has been also used with similar fashion to produce the corresponding thioderivatives of DHPMs (Table 1, entries 9 and 10), which are also of much interest with respect to their biological activities [21]. Using our development methodology, we have also synthesized the target molecules monestrol and nitractin (Table 2, entries 1 and 8) in high yield (95\%) using [Hmim][Tfa] as a catalyst [20]. We have also exploited our proposed methodology for the synthesis of antioxidants (Table 1, Scheme 1, entries 26 and 27) as well as anti-inflammatory via Biginelli reaction, a series of compounds 3-(4,6-disubtituted-2-thioxo-1,2,3,4-tetrahydropy rimidin-5-yl) propanoic acid derivatives (Table 2, entries 29 and 30) were synthesised in high yield and short reaction time.

\section{Conclusion}

In summary, we coupled task specific [Hmim][Tfa] with MW heating and proposed a simple, active and green protocol for the Biginelli reaction. Using this proposed protocol, we synthesized 30 different biologically active dihydropyrimidinones. Low catalyst loading, reduced reaction time and operational simplicity are the main features of this proposed protocol.

\section{REFERENCES}

[1] B. Ganem, "Strategies for Innovation in Multicomponent Reaction Design,” Accounts of Chemical Research, Vol. 42, 2009, pp. 463-472.

[2] L. A. Wessjohann, D. G. Rivera and O. E. Vercillo, "Multiple Multicomponent Macrocyclizations (MiBs): A Strategic Development toward Macrocycle Diversity,” Chem. Rev., Vol. 109, 2009, pp. 796-814.

[3] P. Biginelli and P. Gazz, "Synthesis of 3,4-Dihydropyrimidin-2(1H)-Ones,” Chim Ital, Vol. 23, 1893, pp. 360416.

[4] C. O. Kappe, "Biologically Active Dihydripyrimidones of the Bignelli-Type: A Literature Survey,” European Journal of Medicinal Chemistry, Vol. 35, No. 12, 2000, pp. 1043-1052. doi:10.1016/S0223-5234(00)01189-2

[5] A. Kamal and A. A. Qureshi, "Syntheses of Some Substituted Di-Indolylmethanes in Aqueous Medium at Room Temperature,” Tetrahedron, Vol. 19, No. 4, 1963, pp. 513520. doi:10.1016/S0040-4020(01)98540-0

[6] D. Bandyopadhyay, S. Maldonado and B. K. Banik, "Microwave-Assisted Bismuth Nitrate-Catalyzed Unique Route toward 1,4-Dihydropyridines," Molecules, Vol. 17, No. 3, 2012, pp. 2643-2662.

[7] B. Vijayakumar and G. R. Rao, "Synthesis of 3,4-Dihydropyrimidin-2(1H)-Ones/thiones Using $\mathrm{ZrOCl}_{2} /$ Mont $\mathrm{K} 10$ under Microwave Assisted Solvent-Free Conditions," Journal of Porous Materials, Vol. 19, No. 4, 2012, pp. 491-497.
[8] R. S. Bhosale, S. V. Bhosale, S. V. Bhosale, T. Wangb and P. K. Zubaidhaa, "An Efficient, High Yield Protocol for the One-Pot Synthesis of Dihydropyrimidin-2(1H)-Ones Catalyzed by Iodine,” Tetrahedron Letters, Vol. 45, 2004, pp. 9111-9113.

[9] H. Hazarkhani and B. Karimi, "N-Bromosuccinimide as an Almost Neutral Catalyst for Efficient Synthesis of Dihydropyrimidinones under Microwave Irradiation," Synthesis, Vol. 8, 2004, pp. 1239-1242.

[10] M. Syamla, "Recent Progress in Three-Component Reactions. An Update. 41:1-68. R. Nagarajan and P.T. Perumal, Chem. Lett., 2004, 33, (2009) 288.

[11] N. V. Plechkova and K. R. Seddon, "Applications of Ionic Liquids in the Chemical Industry," Chemical Society Reviews, Vol. 37, 2008, pp. 123-150.

[12] V. Srivastava, "Ionic Liquid Mediated Recyclable Sulphonimide Based Organocatalysis for Aldol Reaction," Central European Journal of Chemistry, Vol. 8, No. 2, 2010, pp. 269-272. doi:10.2478/s11532-009-0140-X

[13] V. Srivastava, “Ionic-Liquid-Mediated MacMillan’s Catalyst for Diels-Alder Reaction,” Journal of Chemistry, Vol. 2013, 2013, pp. 1-9, Article ID: 954094. doi:10.1155/2013/954094

[14] V. Srivastava, “An Improved Protocol for the Preparation of New 1, 2, 3 Triazolium Based Ionic Liquids and Their Application as Solvent for Aldol Reaction," Asymmetric Organocatalysis, Vol. 1, 2012, pp. 2-7. doi:10.2478/asorg-2012-0002

[15] V. Srivastava, "An Improved Protocol for the Aldehyde Olefination Reaction Using (bmim) (NTf2) as Reaction Medium,” Journal of Chemistry, Vol. 2013, 2013, pp. 1-4, Article ID: 439673. doi:10.1155/2013/439673

[16] M. Pucheault and V. Vaultier, “Task Specific Ionic Liquids and Task Specific Onium Salts,” Topics in Current Chemistry, Vol. 290, 2010, pp. 83-126. doi:10.1007/128_2008_33

[17] P. Lidströma, J. Tierneyb, B. Wathey and J. Westmana, "Microwave Assisted Organic Synthesis-A Review," Tetrahedron, Vol. 57, 2001, pp. 9225-9283.

[18] R. M. Palou, "Ionic Liquid and Microwave-Assisted Organic Synthesis: A ‘Green’ and Synergic Couple,” Journal of the Mexican Chemical Society, Vol. 51, 2007, pp. 252-264.

[19] B. L. Hayes, "Recent Advances in Microwave-Assisted Synthesis,” Aldrichimica Acta, Vol. 37, 2004, pp. 66-76.

[20] V. Singh, V. Sapehiyia, V. Srivastava and S. Harika, " $\mathrm{ZrO}_{2}$-Pillared Clay: An Efficient Catalyst for the Solventless Synthesis of Biologically Active Multifunctional Dihydropyrimidinones," Catalysis Communications, Vol. 6, No. 1, 2006, pp. 57-81. doi:10.1016/j.catcom.2004.10.011

[21] T. U. Mayer, T. M. Kapoor, S. J. Haggarty, R. W. King, S. L. Schreiber and T. J. Mitchtison, "Small Molecule Inhibitor of Spindle Bipolarity Identified in a PhenotypeBased Screen,” Science, Vol. 286, No. 5441, 1999, pp. 971-974. doi:10.1126/science.286.5441.971

[22] Suresh and J. S. Sandhu, "Past, Present and Future of the Biginelli Reaction: A Critical Perspective,” ARKIVOC $i$, 2012, pp. 66-133. 